diagnosed with RA and $41.3 \%$ were diagnosed with SpA. The mean disease duration was $6.7 \pm 3.5$ years [1-12] in patients with RA and $6.5 \pm 3$ [1-12] in patients with SpA. The mean Disease Activity Score (DAS28) and Bath Ankylosing Spondylitis Disease Activity Index (BASDAI) were respectively of $4.9 \pm 1.5[1-8]$ and $4.1 \pm 1.8[0-9]$

Therapeutically, 167 patients (56\%) were on Prednisone at a mean daily posology of $8.2 \pm 5.4 \mathrm{mg}$ [ $4-60]$ and $70.3 \%$ on conventional synthetic disease modifying antirheumatic drug (csDMARD) in association with bDMARDs. It was about Tumor Necrosis Factor alpha antibodies (anti TNF a) in $87.9 \%$ of cases, Tocilizumab in $10.4 \%$ of cases and Rituximab in $5 \%$ of cases.

A screening of HBV was performed in 286 patients (96\%). Ag HBs was positive in two cases $(0.7 \%)$, and anti-HBc was positive in 16 cases $(6.4 \%)$ which indicate a prior HBV infection. Fifteen patients $(6 \%)$ were immunized with positive anti HBs. HBV-DNA was measured in 177 cases (66.8\%) and was positive in 15 patients (6\%). $\mathrm{HCV}$ infection was searched in 282 patients (94.6\%) and anti-HCV was negative in all cases.

AST and ALT mean rates were respectively of 18.3 [2-108] and $17.9 \mathrm{UI} / \mathrm{I}$ [2-74]. A perturbation of these liver function tests was observed in 13 patients (4.4\%). Conclusion: Screening for hepatitis B and C were performed respectively in $96 \%$ and $94 \%$ of our Tunisian patients before receiving any bDMARDs. This should be systematic to avoid HBV reactivation which can lead to fulminant hepatic failure with a severe prognosis.

Disclosure of Interests: None declared

DOI: 10.1136/annrheumdis-2020-eular.1083

\section{AB0278 $\quad$ REAL LIFE EXPERIENCE OF DISEASE ACTIVITY AND QUALITY OF LIFE IN PATIENTS TREATED WITH BIOLOGICAL DMARDS VERSUS TOFACITINIB.}

V. Boyadzhieva ${ }^{1}$, N. Stoilov" ${ }^{1}$ E. Kurteva ${ }^{2}$, R. Stoilov ${ }^{1} .{ }^{1}$ University Hospital "St Ivan Rilski", Rheumatology, Sofia, Bulgaria; 'University Hospital "St Ivan Rilski", Laboratory of Clinical Immunology, Sofia, Bulgaria

Background: Assessment of disease activity and quality of life are one of the main indicators for determining the effectiveness of treatment with disease-modifying antirheumatic drugs. In recent years, a new group has entered the market - target synthetic DMARDS, which prove their effectiveness in treating RA comparable to that of biological products.

Objectives: The aim of this study is to evaluate the disease activity and quality of life of patients with rheumatoid arthritis (RA) treated with biological agents in comparison with Tofacitinib (real life data from Bulgarian population) and determine whether or not the benefits of different therapies were sustained over a follow up period of 1 year.

Methods: 164 patients were selected with a mean age $55.34 \pm 16 \mathrm{SD}$ years meeting the 1987 ACR and /or ACR/ EULAR (2010) classification criteria for Rheumatoid arthritis (RA). Patients were arranged according to treatment regimens: Tocilizumab (TCL) 30 patients, Certolizumab (CZP) 16, Golimumab (GOL) 22, Etanercept (ETN) 20, Adalimumab (ADA) 20, Rituximab (RTX) 16, Infliximab (INF) 20, Tofacitinib (TOF) 20. Disease activity and quality of life was the primary concern. Independent joint assessor evaluated 28 joints on baseline, 6th and 12th month's thereafter. CRP was used to measure the inflammatory process.

DAS28-CRP, clinical disease activity index (CDAI) and simplified disease activity index (SDAI)were calculated. On baseline all of the patients' groups had severe disease activity (mean DAS28-CRP > 5.2, mean CDAI > 22, mean SDAI > 26 . The quality of life was evaluated via EQ-5D.

All of the patients were on stable therapy according to the inclusion criteria, and didn't interrupt any of the medications including biological or target synthetic treatment.

Results: Significant clinical improvement and statistically significant reduction in disease activity were observed in patients treated with bDMARDS and tsDMARDS within 6 months ( $p<0.005$ ) of treatment and after 12 months of follow-up $(p=0.039)$. The mean value of DAS28-CRP after one year follow up showed an non-inferior effect of Tofacitnib $(3.04 \pm 0.81)$ in comparison to biological treatment (TCL: $3.07 \pm 0.73$; CZP: $3.06 \pm 0.65$; GOL: $2.49 \pm 0.76$; ETN: $2.85 \pm 0.55$; ADA: $3.15 \pm 0.82$; RTX: $2.90 \pm 0.70$; INF: $3.14 ; \pm 0.61$; TOF: $3.04 \pm 0.81$ ). An improvement was also observed for the 6 to 12 months of follow-up as we did not detect a significant difference in the activity of the disease assessed by CDAl among the different drug groups.

The mean values showing the change of the SDAl over the study period also outline comparable profiles. All of the treatment groups achieved a rapid reduction in disease activity that continued to decrease through the 6 and 12 months period, respectively, as supported by changes in SDAI.The quality of life evaluated with EQ-5D revealed significant improvement on the 6-th month of follow up as well as after 12 th month $(p<0.005)$ without significant difference between the observed groups.

Conclusion: Real-life data show that patients on biological treatment as well as those on Tofacitinib therapy achieve a significant decrease in disease activity after one year of follow-up. This gives us reason to accept the importance of non-inferior effect of jak-inhibitors and their place in treatment of Rheumatoid arthritis.

Disclosure of Interests: Vladimira Boyadzhieva: None declared, Nikolay Stoilov: None declared, Ekaterina Kurteva: None declared, Rumen Stoilov Grant/ research support from: R-Pharm

DOI: 10.1136/annrheumdis-2020-eular.5756

\section{\begin{tabular}{|l|l}
\hline AB0279 & IMPACT OF DISEASE-MODIFYING DRUGS IN SECOND
\end{tabular} BIOLOGICAL TREATMENT SURVIVAL IN PATIENTS WITH RHEUMATOID ARTHRITIS}

A. Briones-Figueroa ${ }^{1}$, M. Tortosa-Cabañas ${ }^{1}$, B. A. Blanco Cáceres ${ }^{1}$, J. Bachiller-Corral ${ }^{1}$, M. Vázquez Díaz ${ }^{1}{ }^{1}$ Ramon y Cajal Hospital, Department of Rheumatology, Madrid, Spain

Background: Several studies have proposed that the immunosenescence of elderly patients with Rheumatoid Arthritis (RA) in treatment with biological therapies could eliminate the need for concomitant immunosuppression with disease-modifying drugs (DMARDs), due to a probable lower production of antidrug antibodies; however, the evidence is limited.

Objectives: To compare the characteristics of patients with RA who started a second biological agent, according to age groups. To analyse second biologica agent survival and its relationship with DMARDs.

Methods: Retrospective, observational and longitudinal study. Patients with RA who started a second biologic between 2000 and 2019, who discontinued a firstline TNF inhibitor, were included. Demographic, clinical and analytical data were obtained. The sample was divided in 2 groups: $<70$ and $\geq 70$ years old. A comparative analysis was performed. Kaplan-Meier curves and Log-rank were used to conduct the survival analysis.

Results: 156 patients were included. $83.3 \%$ were women, with a mean age a the beginning of second biological treatment of $54.64 \pm 13.54$ years. 22 patients $(14.1 \%)$ were $\geq 70$ years. Comparative analysis is detailed in table 1: patients $\geq 70$ years presented a longer time from diagnosis to the start of biological treatment, and a higher prevalence of hypertension and diabetes mellitus. The main cause of withdrawal in this group was adverse events $(46.67 \%)$ while in younge patients was treatment failure $(25.27 \%$ primary failure, $29.66 \%$ secondary failure). The most frequent biological agent in $\geq 70$ years was Rituximab $(27.26 \%)$ while in $<70$ years was Etanercept $(26.12 \%) .126$ patients $(80.8 \%)$ had a DMARD associated. In both groups, Methotrexate was the most frequent (table 2). The second biological agent survival analysis showed that patients who received a DMARD presented a higher survival $[77$ months (55.50-98.55) vs. 51.53 months (41.67-61.40); $\mathrm{p}=0.023$ ]. After conducting a survival analysis in patients whose withdrawal cause was treatment failure, DMARDs use was associated with an increased biological agent survival in patients $<70$ years [103.48 months $(82.28$ $124.68)$ vs. 81.95 months (66.05-97.86); $p=0.037$ ]; but statistical differences were not found in patients $\geq 70$ years [ 117.33 months $(82.15-152.52)$ vs. 65.07 months (40.72-89.42); $p=0.291]$.

Table 1.

\begin{tabular}{lccc}
\hline Variable & $\begin{array}{c}<70 \text { years }=134 \\
\text { (mean } \pm \text { SD or \%) }\end{array}$ & $\begin{array}{c}\geq 70 \text { years }=22 \\
(\text { mean } \pm \text { SD or } \%)\end{array}$ & $p$ \\
\hline Age at diagnosis (years) & $40.5 \pm 12.3$ & $58.8 \pm 8.9$ & $<0.001$ \\
Age at the beginning of the treatment (years) & $51.28 \pm 11.44$ & $75.14 \pm 3.5$ & $<0.001$ \\
Time since diagnosis (years) & $10.65 \pm 8.20$ & $16.27 \pm 9.09$ & 0.003 \\
Women & $113(84.33 \%)$ & $17(77.27 \%)$ & 0.373 \\
Smokers & $29(21.64 \%)$ & $2(9.09 \%)$ & 0.320 \\
Rheumatoid factor positive & $109(81.34 \%)$ & $17(77.27 \%)$ & 0.770 \\
Anti-CCP positive & $114(90.48 \%)$ & $14(82.35 \%)$ & 0.390 \\
Erosions & $92(70.23 \%)$ & $16(76.19 \%)$ & 0.576 \\
Arterial hypertension & $28(21.37 \%)$ & $14(66.67 \%)$ & $<0.001$ \\
Diabetes mellitus & $3(2.24 \%)$ & $4(18.18 \%)$ & $<0.001$ \\
Retirement & $91(67.91 \%)$ & $15(68.18 \%)$ & 0.980 \\
Infections & $10(7.46 \%)$ & $3(13.64 \%)$ & 0.397 \\
Second biological agent withdrawal cause & & & \\
Primary failure & $23(25.27 \%)$ & $3(20 \%)$ & 0.242 \\
Secondary failure & $27(29.66 \%)$ & $3(20 \%)$ & \\
Adverse events & $25(27.47 \%)$ & $7(46.67 \%)$ & \\
Remission & $2(2.20 \%)$ & $0(0 \%)$ & \\
Exitus & $3(3.30 \%)$ & $0(0 \%)$ & \\
Neoplasia & $3(3.30 \%)$ & $0(0 \%)$ & \\
\hline
\end{tabular}


Table 2.

\begin{tabular}{lccc}
\hline Disease-modifying drug & $<70$ years old & $\geq 70$ years old & $p$ \\
\hline Methotrexate & $72(53.73 \%)$ & $9(40.91 \%)$ & 0.667 \\
Leflunomide & $22(16.42 \%)$ & $5(22.73 \%)$ & \\
Sulfasalazine & $2(1.49 \%)$ & $1(4.55 \%)$ & \\
Hydroxychloroquine & $6(4.48 \%)$ & $0(0 \%)$ & \\
At least two of the above & $7(5.22 \%)$ & $1(4.55 \%)$ & \\
\hline
\end{tabular}

Conclusion: DMARD concomitant treatment has been related to a higher second biological treatment survival. This beneficial effect was not observed in RA patients $\geq 70$ years of age whose second biological agent withdrawal cause was failure. In this age group, withdrawal related to adverse events was more frequent. References:

[1] Kalden JR, Schulze-Koops H. Immunogenicity and loss of response to TNF inhibitors: implications for rheumatoid arthritis treatment. Nature reviews Rheumatology. 2017;13(12):707-718.

Disclosure of Interests: None declared

DOI: 10.1136/annrheumdis-2020-eular.4979

\section{AB0280 SURVIVAL ANALYSIS ON SECOND BIOLOGIC THERAPY IN PATIENTS WITH RHEUMATOID ARTHRITIS OLDER THAN 65 YEARS}

A. Briones-Figueroa ${ }^{1}$, M. Tortosa-Cabañas ${ }^{1}$, B. A. Blanco Cáceres ${ }^{1}$, J. L. Morell Hita $^{1}$, J. Bachiller-Corral ${ }^{1}$, M. Vázquez Díaz ${ }^{1}{ }^{1}$ Ramón y Cajal University Hospital, Department of Rheumatology, Madrid, Spain

Background: Patients with Rheumatoid Arthritis (RA) $\geq 65$ years old constitute an important and not very well studied group. Even though the course of the disease may be similar to that of younger patients, treatment is usually less aggressive given the limited information on efficacy, especially of biological treatments, in this age group.

Objectives: To describe the characteristics of patients with RA $\geq 65$ years old who started a second biological agent. To compare the survival of this second-line treatment between patients $\geq 65$ and $<65$ years old.

Methods: Retrospective, observational and longitudinal study. Patients diagnosed of RA, who started a second biological agent between 2000 and 2019 , who discontinued a first-line TNF inhibitor, were included. Demographic, clinical and analytical data were obtained. The sample was divided in 2 groups: $<65$ and $\geq 65$ years old. Kaplan Meier and Log-rank survival analysis were performed, as well as Cox regression to identify related factors.

Results: 157 patients were identified, $42(26.8 \%)$ were $\geq 65$ years old. In this group, $73.8 \%$ were women, with a mean age at the beginning of second biological treatment of $71.43 \pm 4.76$ years. Demographic and clinical data of $\geq 65$ years old patients are shown in the table. The most frequent second biological agent was Rituximab (23.8\%), followed by Adalimumab (21.4\%) and Tocilizumab (19\%). $76.2 \%$ of patients had a disease-modifying drug associated, being Methotrexate the most frequent $(45.2 \%)$. Discontinuation of second biological agent occurred in 30 patients $(71.42 \%) \geq 65$ years old, which is similar to the percentage found in patients $<65$ years old $(66.96 \% ; p=0.70)$. The main causes of withdrawal of second-line agent in patients $\geq 65$ years were adverse effects $(23.8 \%)$ and secondary failure $(23.8 \%)$, whereas in $<65$ years were primary and secondary failure (18.3\% in both). Infections were more frequent in patients $\geq 65$ years $(14.3 \%$ ) in comparison with patients $<65$ years $(6.1 \%)$. In the survival analysis of the second biological agent, patients $\geq 65$ years presented a median survival of 45 months (IC-95\%=14.10-75.90); while patients $<65$ years had a median survival of 47 months (IC- $95 \%=29.55$ $64.46)$, without statistically significant differences $(p=0.803)$ (See Figure). Among elderly patients no statistically significant differences were found after comparison of survival curves in the subgroups: $65-69,70-74$ and $\geq 75$ years. Rituximab presented a higher survival rate in patients $\geq 65$ years $(84.3$ months; $p<0.001)$, followed by Abatacept (58.5 months). Smoking (HR=13.96; IC- $95 \%=2.12-91.93)$, erosions $(H R=7.04 ; I C-95 \%=1.05-47.31)$ and diabetes mellitus (HR=13.37; IC-95\%=1.25-143.46) were identified as risk factors for discontinuation of second biologic agent.

Conclusion: The survival of second biological agent after the failure of a first TNF inhibitor in patients $\geq 65$ years is similar to the survival in younger patients, although there was a higher percentage of adverse effects in the first group. Rituximab and Abatacept showed a higher survival in patients $\geq 65$ years. Smoking, erosions and diabetes mellitus were associated with an increased risk for the withdrawal of the second-line biological therapy.

References:

[1] Richter M, Matteson E, DavisIII J, Achenbach S, Crowson C. Comparison of Biologic Discontinuation in Patients With Elderly-Onset Versus Younger-Onset Rheumatoid Arthritis. ACR Open Rheumatology. 2019; 1(10): 627-631.

\section{Variables}

$\mathrm{n}=42($ mean $\pm \mathrm{SD}$ or $\%)$

Age at diagnosis (years)

Age at the beginning of the treatment (years)

Women

Smokers

$\mathrm{RF}(+)$

ACPA (+)

Erosion

Arterial hypertension

Diabetes mellitus

Dyslipidemia

Disease-modifying drug

Methotrexate

Leflunomide

Sulfasalazine

Hydroxychloroquine

Infections

Respiratory infection

Skin/Soft tissues infection

Herpes zoster

$56.48 \pm 9.94$

$71.43 \pm 4.76$

$31(73.8 \%)$

$11(26.2 \%)$

$35(83.3 \%)$

$30(71.4 \%)$

$21(50 \%)$

$4(9.5 \%)$

$11(26.2 \%)$

$19(45.2 \%)$

$10(23.8 \%)$

$1(2.4 \%)$

$0(0 \%)$

$3(7.1 \%)$

$2(4.8 \%)$

$1(2.4 \%)$

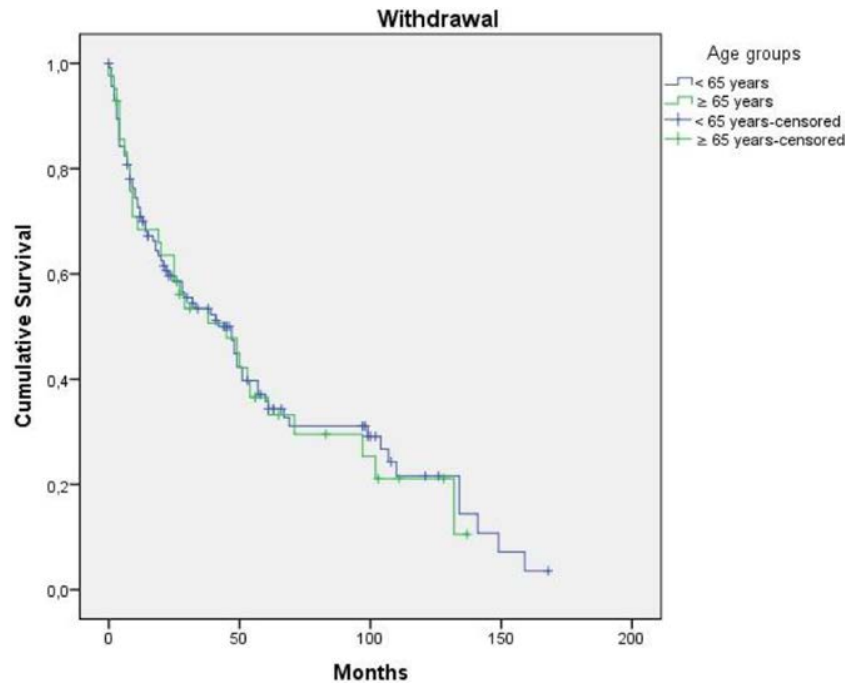

Disclosure of Interests: None declared

DOI: 10.1136/annrheumdis-2020-eular.4911

\section{AB0281 \\ SAFETY AND RETENTION RATE AFTER SWITCHING FROM ETANERCEPT ORIGINATOR (ETN) TO ETANERCEPT BIOSIMILAR (SB4) IN INFLAMMATORY JOINT DISEASES: DATA FROM REAL LIFE.}

C. Bruni ${ }^{1}$, S. Gentileschi ${ }^{2}$, M. Capassoni ${ }^{1}$, G. Pacini ${ }^{1}$, M. Bardelli ${ }^{2}$, C. Baldi ${ }^{2}$ L. Tofani ${ }^{1}$, L. Cometi ${ }^{1}$, F. Nacci' ${ }^{1}$,F. Bartoli ${ }^{3}$, G. Fiori ${ }^{3}$, L. Cantarini ${ }^{2}$,

S. Guiducci ${ }^{1,3}$, B. Frediani ${ }^{2}$, M. Matucci-Cerinic ${ }^{1,3} .{ }^{1}$ University of Florence, Faculty of Medicine, Experimental and Clinical Medicine, Division of Rheumatology, Firenze, Italy; ${ }^{2}$ University of Siena, Medical Sciences, Surgery and Neurosciences, Rheumatology Unit, Siena, Italy; ${ }^{3}$ Careggi University Hospital, Rheumatology, Firenze, Italy

Background: SB4 in now commonly used in the treatment of inflammatory joint diseases, with evidence of efficacy and persistence up to 12 months from switching in both randomized controlled trials in Rheumatoid Arthritis (RA), Psoriatic Arthritis (PsA) and Ankylosing Spondylitis (AS).

Objectives: we investigated the safety and retention rate of SB4 at 6,12 and 18 months after switching from ETN in two rheumatology departments in our region. Methods: adult patients with RA, PsA, AS, Juvenile Idiopathic Arthritis (JIA) and other rheumatic diseases treated with ETN for at least 6 months, switched to SB4 in stable clinical conditions, were eligible for this retrospective evaluation. Data on adverse events (in particular infectious events), loss of efficacy (articular, cutaneous, ocular or intestinal disease re-activation) and persistence on treatment were collected since latest available follow-up. Retention rate, reason for discontinuation and subsequent management data were collected at $6,12,18$ months. Results: 220 patients (142 females, mean age 58+-7 years, disease duration 12+-4 years, ETN duration 7+-4 years) were enrolled, with median follow up of 12.1 (9.7-15.8) months duration; ETN was used in different biologic DMARDs treatment lines (first $76.8 \%$, second $17.7 \%$, third $3.2 \%$, fourth $2.3 \%$ ). Study 